An unusual finding at screening colonoscopy: polypoid follicular lymphoma with marginal zone differentiation

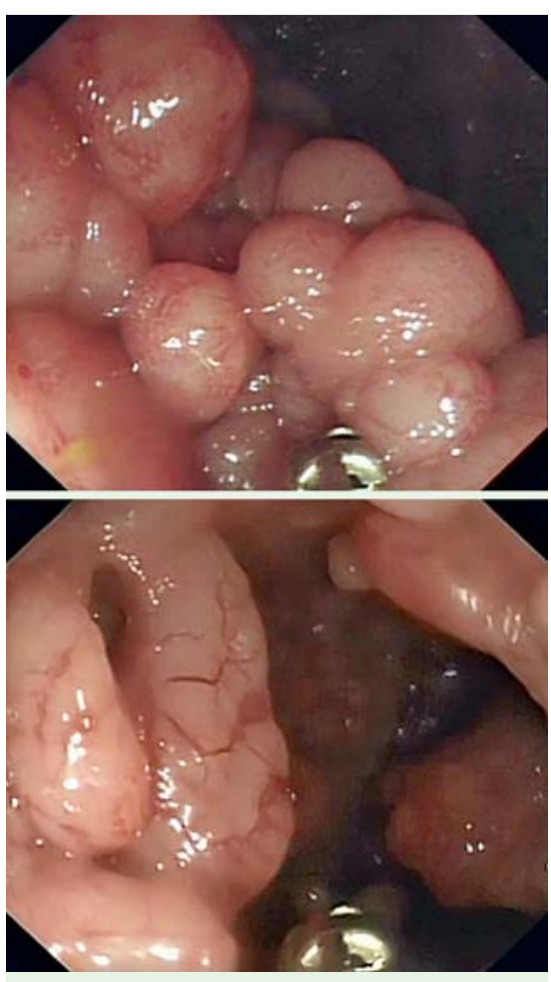

Fig. 1 Endoscopic views of the cecum showing unusual polypoid lymphomatous lesions.

An asymptomatic 60-year-old man underwent screening colonoscopy after positive fecal occult blood testing (FOBT) during participation in the UK national bowel cancer screening program. Colonoscopy revealed clusters of sessile polypoid masses involving the rectum, sigmoid colon, cecum, and terminal ileum ( $\bullet$ Fig. 1 , - Video 1).

The largest lesion affected the cecum and measured about $7 \mathrm{~cm}$ across. Histopathological examination demonstrated abnormal lymphoid proliferation, consisting of irregular follicles with mantles and abnormal germinal centers. Immunohistochemistry showed predominant B-cell presence. Staining for CD10 and BCL6 was negative, with $\mathrm{K}$ light-chain restriction in nodule peripheries: these features favored a diagnosis of extranodal B-cell lymphoma, marginal zone subtype. Staging bone-

\section{Video 1}

Endoscopic views of cecum showing unusual lymphomatous polypoid lesions.

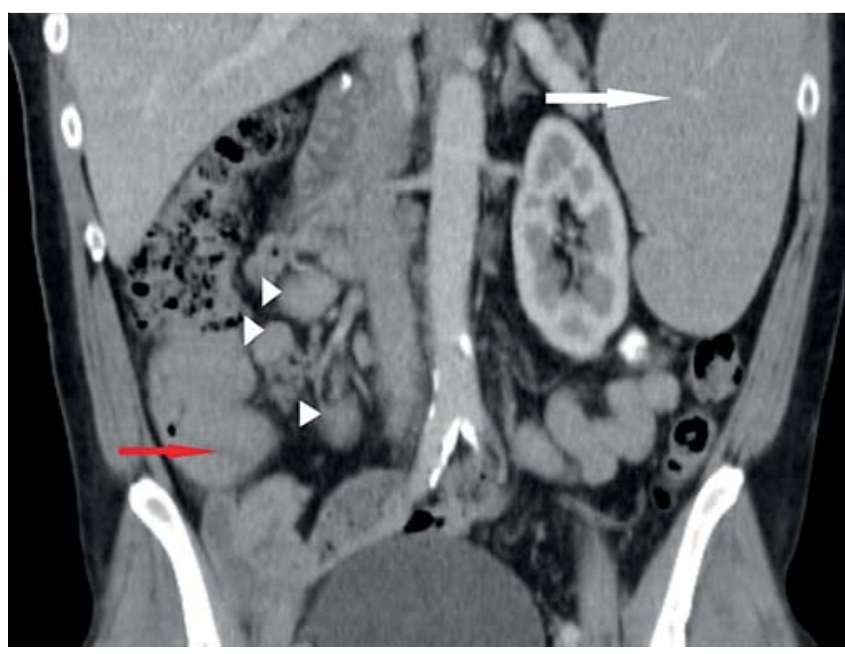

Fig. 2 Computed tomography (CT) scan of the abdomen, coronal view, showing thickened cecum (red arrow), mesenteric nodes (white arrowheads), and enlarged spleen (white arrow).

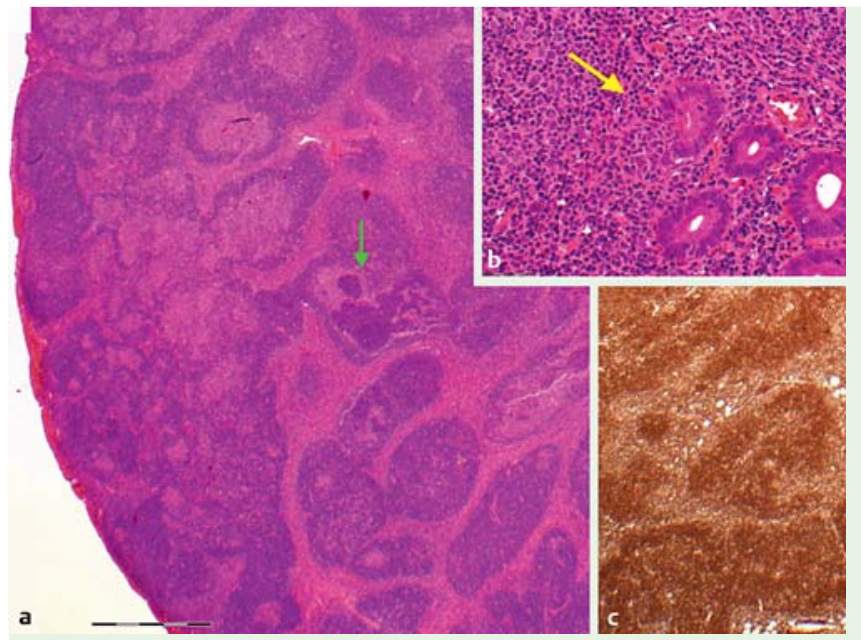

Fig. 3 Histopathology of the colonic lesions. a The follicle in the center (green arrow) appears to be colonized by smaller cells from outside, a feature supporting marginal zone differentiation (hematoxylin and eosin, scale bar $1 \mathrm{~mm}$ ). b Destructive lymphoepithelial lesion, another feature of marginal zone differentiation (yellow arrow) (hematoxylin and eosin stain, scale bar $50 \mu \mathrm{m}$ ). c All follicular B cells stained positively for BCL2, characteristic of follicular lymphoma (same magnification as in part a). marrow biopsy showed 25\% B-cell lymphoma involvement and cytogenetics of marrow cells highlighted IgH-BCL2 translocation, typical of follicular lymphoma. Computed tomography demonstrated diffuse lymphadenopathy, splenomegaly, and thickening of the cecum associated with large mesenteric nodes ( $\bullet$ Fig. 2).

Upper gastrointestinal endoscopy and biopsies were unremarkable, and Helicobacter pylori tests were negative. Immunohistochemistry of an axillary node biopsy confirmed predominant B-cell infiltration and, unlike the lower gastrointestinal histopathology, showed focal positivity for CD10 and BCL6, favoring the diagnosis of follicular lymphoma. Immunohistochemistry of further, larger colonic biopsies unified a final diagnosis of follicular lymphoma with marginal zone differentiation ( $\bullet$ Fig.3) and confirmed common clonal origin.

The overall clinical diagnosis fulfilled the criteria [1] for follicular lymphoma, stage 
IVA [2]. Immuno-chemotherapy was well tolerated and restaging after cycles 3 and 6 confirmed a good response.

Presentation of follicular lymphoma through detection of large, asymptomatic colonic lesions is very unusual [3-5]. Marginal zone differentiation in follicular lymphoma is also atypical and is associated with shorter survival, independent of stage and treatment [6]. Another remarkable feature here is the persistent silence of the follicular lymphoma, which, despite its advanced stage, was only unmasked by an FOBT at bowel cancer screening. A colonic presentation of follicular lymphoma should therefore prompt clinicians to cascade a thorough search for systemic disease.

Endoscopy_UCTN_Code_CCL_1AD_2AC

Competing interests: None
E. J. Despott ${ }^{1}$, P. J. Tadrous ${ }^{2}$, K. N. Naresh ${ }^{3}$, A. Savio ${ }^{2}$, M. Marshall ${ }^{4}$, B. P. Saunders ${ }^{1}$

1 Wolfson Unit for Endoscopy, St Mark's Hospital and Academic Institute, Imperial College London, UK

2 Department of Pathology, North West London Hospitals NHS Trust, London, UK

3 Department of Academic Histopathology, Hammersmith Hospital, Imperial College Healthcare NHS Trust, London, UK

4 Department of Radiology, NWLH NHS Trust, Watford Road, Harrow, London, UK

\section{References}

1 Jaffe ES, Harris NL, Stein H et al. Classification of lymphoid neoplasms: the microscope as a tool for disease discovery. Blood 2008; 112 : 4384-4399

2 Carbone PP, Kaplan HS, Musshoff K et al. Report of the Committee on Hodgkin's Disease Staging Classification. Cancer Res 1971; 31: 1860-1861

3 Hiraide T, Shoji T, Higashi Y et al. Extranodal multiple polypoid follicular lymphoma of the sigmoid colon. Gastrointest Endosc 2011; 73 (1): $182-184$
4 Dalal L. Primary multifocal non-Hodgkin lymphoma of the colon successfully treated with chemotherapy. Gastrointest Endosc 2008; 68: 1005-1006

5 Kakati BR, Krishna SG, Dang SM et al. Colonic perforation following polypectomy of a gastrointestinal follicular lymphoma masquerading as a colon polyp. J Gastrointest Cancer 2010 October 5 [Epub ahead of print]

6 Nathwani BN, Anderson JR, Armitage JO et al. Clinical significance of follicular lymphoma with monocytoid B cells. Non-Hodgkin's lymphoma classification project. Hum Pathol 1999; 30: 263-268

Bibliography

DOI $10.1055 / \mathrm{s}-0030-1256599$

Endoscopy 2011; 43: E266 - E267

(c) Georg Thieme Verlag KG Stuttgart · New York . ISSN 0013-726X

Corresponding author

E. J. Despott

Wolfson Unit for Endoscopy

St Mark's Hospital and Academic Institute

Imperial College London

Watford Road

London HA1 3UJ

United Kingdom

Fax: +44-(0)-208-423-3588

edespott@doctors.org.uk 